\title{
A review of alcohol use in the transgender population
}

\section{Fátima Urzal ${ }^{1}$, Inês Donas-Boto' ${ }^{1}$, Sónia Farinha Silva²}

1 - Department of Psychiatry and Mental Health, Centro Hospitalar de Lisboa Ocidental

2 - Department of Psychiatry, Unidade de Saúde Local do Baixo Alentejo

\section{Objectives}

The authors intend to characterize the patterns of alcohol consumption in the transgender population and to discuss its determinants and consequences.

\section{Background and aims}

Gender minority populations stand at an increased risk for substance abuse, particularly alcohol misuse, having been recognized as priority groups for intervention. However, few studies have comprehensively reviewed the recent prolific evidence on this subject.

\section{Materials and methods}

Pubmed database was searched from 2009 to 2019 using the terms "transgender" and "alcohol".

\section{Results}

The search returned 191 studies which met the inclusion criteria.

Both social expectations and physiological differences weigh in on the definition of alcohol misuse criteria according to gender. ${ }^{1}$ However, this is a difficult definition for transgender people, since physiological sex characteristics can shift upon gender transition, but the effects on the metabolization of alcohol are mostly unknown. ${ }^{1}$

Alcohol misuse is consistently higher in transgender people compared with the general population, particularly in early to middle adulthood ${ }^{2,3}$, and namely through:

\section{Binge drinking}

\section{Drinking to intoxication}

Lifetime alcohol use disorder

Past-year alcohol use disorder
$4,5,6,7,8$

\section{7 to $61 \%$}

25 to $58 \%$

\begin{tabular}{ll}
7 to $61 \%$ & $4,9,10,11$ \\
\hline 25 to $58 \%$ &
\end{tabular}

12

\section{$26 \%$}

\section{$11 \%$}

Some studies indicate alcohol consumption is heavier in transgender women. ${ }^{14,15}$

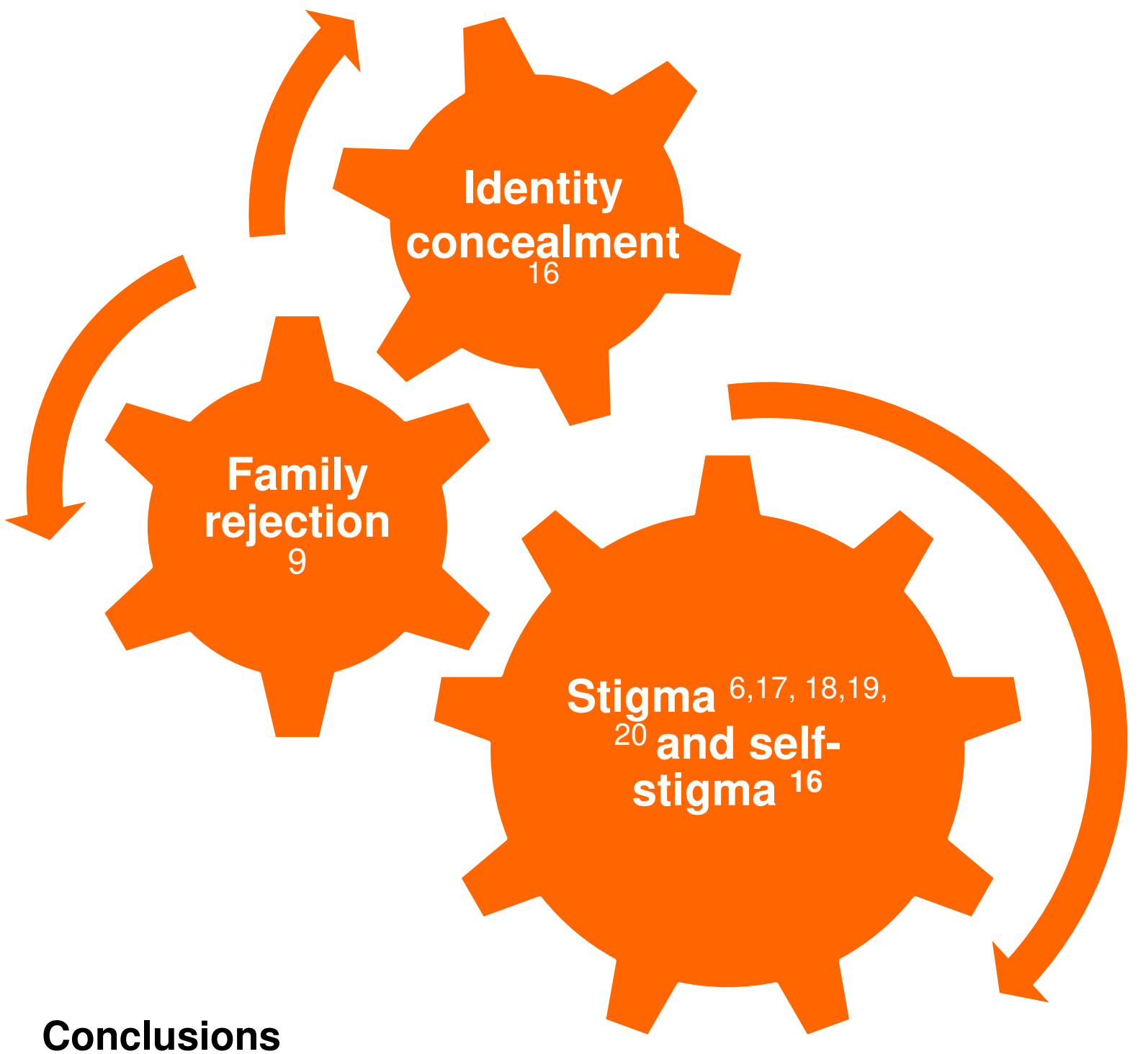

Alcohol misuse seems to be at least partially explained by maladaptive coping behaviors to deal with discrimination and stigma (picture 1).

The consumption of alcohol is associated with high risk sexual behaviors and increased risk of victimization, namely sexual and physical assault. 4,21

Most studies did not specify transgender status or consider the distinctions between sex and gender in study design and data analysis. Furthermore, the authors found no global intervention programmes designed for this particular population.

\section{Conclusions}

The increased risk for alcohol misuse in the transgender population has important psychosocial determinants. Intervention strategies should target discrimination and consider the higher risk in younger people. Future studies must improve the quality of data concerning sex and gender. 